\title{
Trends
}

Advances in research and medical practices have made significant inroads towards the treatment of diseases at the single patient level. This paradigm, precision medicine, holds the promise of reducing adverse effects, improving preventative care, and reducing costs by tailoring individual treatment based on highly detailed diagnostics.

Humans harbor trillions of microbes, termed the microbiome, which is now being appreciated as being a hugely substantial facet of health. Immune, metabolic, neurological, and other processes impact and are impacted by the microbiome.

The microbiome not only is a significant factor in health, but it is one that can be both readily assayed through DNA sequencing and directly modified by various targeted interventions. Therefore, the currently genetic information dominated field of precision medicine would be greatly enhanced by the introduction of the microbiome. 


\section{Introducing the Microbiome into Precision Medicine}

2 Thomas M. Kuntz ${ }^{1,2} \&$ Jack A. Gilbert ${ }^{2,3,4}$

$3{ }^{1}$ Department of Chemistry, University of Chicago, 929 East 57th Street, Chicago, Illinois

$4 \quad 60637$, USA

52 The Microbiome Center, Department of Surgery, University of Chicago, $5841 \mathrm{~S}$

6 Maryland Ave, Chicago, IL, U.S.A

$7 \quad{ }^{3}$ Biosciences Division (BIO), Argonne National Laboratory, 9700 South Cass Avenue,

8 Argonne, IL, U.S.A

$9 \quad{ }^{4}$ Marine Biological Laboratory, Woods Hole, MA, U.S.A

10 *Correspondence: gilbertjack@uchicago.edu (J.A. Gilbert)

12 Abstract

13 Understanding how individual people respond to medical therapy is a key facet of

14 improving the odd-ratio that interventions will have a positive impact. Reducing the non-

15 responder rate for an intervention or reducing complications associated with a particular

16 treatment or surgery is the next stage of medical advance. The Precision Medicine

17 Initiative, launched in January 2015, set the stage for enhanced collaboration between

18 researchers and medical professionals to develop next-generation techniques to aid

19 patient treatment and recovery, and increased the opportunities for impactful preemptive

20 care. The microbiome plays a crucial role in health and disease, as it influences

21 endocrinology, physiology and even neurology, altering the outcome of many different

22 disease states, and it augments drug responses and tolerance. We review the implications 
23 of the microbiome on precision health initiatives and highlight excellent examples, 24 whereby precision microbiome health has been implemented.

25 


\section{Introduction to Precision Medicine}

27 The sequencing of the human genome [1] in 2001 fostered advances in both our

28 understanding of the genomic basis of disease and in the DNA sequencing technologies

29 required to bring the results of this understanding to patients. This is often referred to as

30 precision genomic medicine, which utilizes a patient's individual genome to inform

31 treatment and care, based on known genomic markers for disease [2]. The broader,

32 inclusive field of precision medicine couples a person's treatment with what is known

33 about their population, life style, and medical history, by matching clinical data and

34 genetic biomarkers. Since the genome is sometimes conceptualized as the core of human

35 individuality, at least in terms of disease, the broader field of precision medicine is often

36 conflated with genomic medicine. Precision medicine, however, includes aspects

37 downstream from the genome, including gene expression and protein expression as well

38 as metabolic markers. Nonetheless, genomic information is the most commonly used and

39 has had great successes [3]. Cancer treatment in particular has been revolutionized by

40 genomic medicine [4], which exemplifies that despite difficulties in implementing

41 precision medicine, it is a deeply important development. In particular, achieving the

42 goals of precision medicine, including diagnosing disease more accurately and reducing

43 the relative risk of treatments, side effects, and non-responses to medications, will

44 revolutionize both treatment courses — ideally at the single patient level [5] — and the

45 structuring of medical care and costs, moving towards cheaper, preventative focused

46 medicine.

48 The Microbiome as a Precision Medicine Frontier 
49 In this review we focus on a more recent but in many ways analogous development, that

50 of introducing the microbiome into precision medicine. The human microbiome is the

51 "the ecological community of commensal, symbiotic, and pathogenic microorganisms

52 that literally share our body space" [6]. These microorganisms, mainly bacteria, fungi,

53 archaea, and viruses in the gastrointestinal tract, are slightly more abundant than the

54 human cells in the body, leading some to classify them as an newly discovered organ [7].

55 It is important to note however that the microbiome is compositionally and

56 spatiotemporally far more fluid and mutable than human cells and organs. Therefore, the

57 microbial "organ" may be better described as a "cloud" of genetic information accessory

58 to the stable human genome [8]. Certainly, the influences of the microbiome on our

59 physiology are significant and multitudinous, affecting immunology [9], neurology

$60[10,11]$, endocrinology [12], and, importantly for precision medicine, disease states and

61 clinical outcomes. Because microbiome science is a nascent but quickly developing field,

62 additional important functions of the microbiome are likely still to be discovered. These

63 discoveries are driven by similar sequencing technology as that which has enabled

64 personal genomics, and this technology is decreasing rapidly in price [13], so much so

65 that personal microbiome sequencing is already available to the consumer (e.g. American

66 Gut - americangut.org; uBiome - ubiome.com). Furthermore, the well-developed analysis

67 and statistical techniques of genomic medicine have commonalities with microbiome

68 analysis. Since microbiome states are highly individual even between co-raised identical

69 twins [14], but can be rapidly changed [15] (unlike genetics), there is a profound

70 opportunity for individualized treatments. However, the microbiome, like any ecosystem

71 is also profoundly complex, and so the goals of precision microbial medicine require 
72 considerably more research before they are appropriately realized [16]. Nonetheless, the

73 microbiome, as we shall exemplify here, is primed and ready for precision medicine, and

74 therefore the clinical application of this new therapeutic area is on the immediate horizon.

75 Various complementary routes of assaying and modifying the microbiome have been

76 proposed and tentatively utilized towards this end; these will be laid out here in the

77 following text as well as diagrammatically (Figure 1).

\section{Review of Microbiome Analysis Techniques}

80 How then could microbiome precision medicine be implemented? Currently two 81 complementary analyses, both beginning with the extraction of microbial genomic DNA, 82 are standard in the field: $16 \mathrm{~S}$ rRNA sequencing and shotgun metagenomics. The $16 \mathrm{~S}$

83 rRNA gene has both highly conserved regions, allowing for the usage of extremely

84 bacterially nonspecific primers, and "hypervariable" regions, where base pair differences

85 can often provide species level identification [17]. Thus, 16S rRNA amplicon sequencing

86 provides a robust tool for identification as well as classification and even discovery of

87 bacteria [18]. A typical 16S rRNA study utilizes the differences in observed communities

88 of bacteria between differing samples to obtain statistically significant correlations

89 between bacterial composition and sample description, for example to identify

90 differences in the gut microbiomes of children born to obese mothers [19]. These studies

91 have led to key insights into the human microbiome. While historically the majority of

92 biomedical research on bacteria has focused on eliminating pathogens, many bacteria as

93 well as communities of bacteria are important in both health and disease [6]. Though

94 identifying causative bacteria in disease states will be an important facet of precision 
95 medicine, understanding the overall ecology of the microbiome may be equally or even

96 more vital.

97

98 Therefore, to go beyond bacterial identification and subsequent, limited patient

99 stratification, it will be essential to understand the functional potential of the microbiome.

100 Shotgun metagenomics enables the researcher to understand this function potential

101 through analysis of the complete genomic repertoire of the community, by sequencing

102 DNA extracted from that community, rather than relying on amplification of a marker

103 gene. Taxonomy can still be determined from signature genes (including 16S rRNA), but

104 it is also possible to assign phylogeny of the functional genes by comparing the DNA

105 sequence against a library of genomes from close relatives [20]. In addition,

106 metagenomics enables the assembly of genomes from organisms in the microbiome that

107 are resistant to culture, providing a higher resolution exploration of the taxa associated

108 with each person [21]. This enables us to determine the metabolic and signaling capacity

109 of each taxon, to determine how it will interact with the rest of the body [22]. This clearly

110 makes metagenomics of great interest for the development of precision medicine;

111 however, one must be aware of the challenges this technique presents. Metagenomic

112 studies are necessarily more expensive and computationally complex than 16S rRNA

113 based studies. Possible contamination from undesired DNA and biases of analyses

114 towards culturable organisms [23] further complicate matters. Ultimately metagenomics

115 is an extremely useful tool, but the application of this technology to precision medicine

116 will require a better understanding of the implications of these limitations, especially

117 when scaling up to treatments of large patient populations. 
119 Notably, both 16S sequencing and shotgun metagenomics are currently somewhat blunt 120 tools, especially when describing the fluid nature of the microbiome. Evolution of

121 microorganisms, horizontal transfer of genes, and subtleties in the characterization into

122 types of microbiomes [24] problematize the microbiome snapshot style data often

123 acquired. As sequencing costs continue to decrease, however, scientists can sample more

124 densely in time to capture previously unobservable subtleties in microbial interactions

125 and utilize time series techniques to uncover dynamic ecological phenomena [25].

126 Additionally, the gut microbiome is known to be spatially inhomogeneous, in ways that

127 influence function and disease states [26]. This limitation too might be surpassed in the 128 near future, owing to emerging sampling techniques and protocols (e.g., laser 129 microdissection of colonic crypt mucus [27]).

\section{Avenues Towards Microbiome-Based Precision Therapies}

\section{Microbiome-xenobiotic interactions}

133 That gene polymorphisms can drive changes in drug metabolism has been known for

134 some time; it was noted as early as 1957 that atypical forms of serum cholinesterase led

135 to potentially fatal reactions to certain anesthetics [28]. This and other adverse drug 136 reactions are estimated to cost from 30 to 130 billion dollars in the USA annually [29,30]

137 and are a significant source of patient non-compliance and therapy failure [31]. Reducing 138 these adverse reactions is a primary goal of precision medicine. While some interactions 139 are idiosyncratic, a recent survey of adverse drug events observed that about $35 \%$ of 140 these events were drug-gene or drug-drug-gene interactions involving cytochrome P450 
141 oxidase (CYP) variants [32]. CYPs are generally considered the body's innate and

142 primary general purpose drug metabolizers; they are involved in about $75 \%$ of total 143 human drug modification [33].

145 However, microbial metabolism in the gut is also a significant factor in 146 biotransformation, especially for low solubility, low permeability compounds [34]. 147 Currently, more than 60 drugs have been identified to have microbiome interactions 148 according to the PharmacoMicrobiomics database [35], and given the vast number of 149 possible unique microbial metabolic transformations [36], many more interactions are 150 likely to be discovered compared with the apparently relatively limited number of human 151 genetic interactions. The plasticity of the microbiome may make these interactions 152 dynamic, necessitating precision medicine that is not only patient specific but temporarily 153 appropriate [37]. Importantly, the primary forms of xenobiotic metabolism are different 154 between human and bacterial cells: oxidation and conjugation dominate in the former 155 case, reduction and hydrolysis in the latter [34]. Metabolism of drugs is actually a key 156 component of many therapies; so-called "prodrugs" are essentially drugs that will be 157 metabolized into a pharmacologically active drug after consumption. Therefore, 158 production of active drug metabolites from prodrugs is sometimes dependent on the 159 microbiome, with the possibility to either improve or worsen outcomes [38]. This often 160 manifests as a modulation of bioavailability to the human, an important consideration for 161 prediction of appropriate dosing in precision medicine. Efficacy and side effects are also 162 altered directly by microbial metabolism. For example, acetaminophen toxicity shows 163 substantial variability within a given human population [39], and the microbiome has 
164 been identified as playing a role in this variability. Members of the genus Clostridium, as

165 well as other bacteria can produce $p$-cresol, which competes as a substrate for SULT1A1

166 (a human liver enzyme) with acetaminophen [40]. A reduction in the breakdown of

167 acetaminophen by SULT1A1 causes a build-up of NAPQI, which leads to hepatotoxicity.

168 This general pattern of competition between bacterial metabolites and drugs for human

169 enzyme modification constitutes a major challenge in pharmacology [41]. Directly

170 harmful substances can also be formed by microbiota, as is the case in bacterial $\beta$ -

171 Glucuronidase mediated diarrhea in response to an antitumor camphothecin derivative

172 [42]. Strikingly, in some cases even strain level differences can lead to altered

173 metabolism, such as inactivation of digoxin by a non-universal E. lenta gene. Digoxin has

174 a narrow therapeutic window, and thus a wrong dosage could lead to significant toxicity,

175 highlighting the need for further study of metagenomic diagnostics and insights to

176 adverse outcomes [43].

177

178 Furthermore, alternative mechanisms for xenobiotic-microbiome interaction including

179 immune $[9,44,45]$ and endocrine [12] modulation by bacteria are known to exist,

180 complicating and enlarging the pool of possible drug-microbiome interactions. Lastly,

181 there are possible reciprocal relations: drugs may both be altered by the microbiome and

182 alter the microbiome. For example, antipsychotic medication has been shown to both

183 alter the microbiome and have microbiome-dependent side effects [46]. While this

184 greatly complicates endeavors to understand microbiota-xenobiotic interactions, it also

185 points towards a different microbiome driven approach to precision medicine: directly

186 targeting the microbiome for clinical results. 


\section{Targeting the microbiome}

189 It is clear that medication is already utilized to have a direct effect on the microbiome;

190 one needs to look no further than antibiotics. While these drugs are utilized for the

191 eradication of pathogenic bacteria, they have widespread effects on the microbiome,

192 possibly leading to adverse outcomes. Secondary infections caused by antibiotics are well

193 known, most saliently Clostridium difficile [47], but it is often less appreciated that

194 antibiotics can have side effects on the human, for instance fluoroquinolone associated

195 cardiotoxic [48] and neuropsychiatric [49] reactions. Importantly, consequences of

196 antibiotic usage, such as reduction of inflammation, are possibly not only human off-

197 target drug effects, but also unintended consequences of microbial community disruption

198 [50]. Studies using mouse models suggest that stress induced increases in circulating

199 cytokines were abrogated by broad-spectrum antibiotic treatment [51]. Furthermore,

200 these types of interactions are not limited to drugs classified as antibiotics; many other

201 drugs have antibiotic and other microbial community structure and function modulating

202 properties that are beginning to be appreciated [52,53]. While many of these

203 perturbations to the microbiome are associated with poorer outcomes, some drugs may

204 derive some or all of their beneficial qualities from alteration of the microbiome, thus

205 they could be considered a form of discriminatory antibiotic.

207 A precision medicine therapy that leverages microbial community structural modulation

208 could have beneficial clinical impact. Certainly if pathogen-specific antibiotics were

209 developed, the odds ratio could be greatly increased compared to traditional antibiotics. A 
210 clear approach is to design a species-specific enzyme inhibitor or other antimicrobial

211 molecules. For example, a Streptococcus mutans-targeted drug based on the fusion of a

212 species-specific targeting peptide domain with a wide-spectrum antimicrobial peptide

213 domain has already been developed [54]. However, the bacterial community was also

214 altered when using this peptide, despite its high specificity [55]. This is likely because the

215 environment of Streptococcus mutans, the oral microbiome, presents significant

216 structural and functional complexity [56]. It has been suggested that targeted antibiotics

217 may shift the microbiome into a healthier state, but of course there is also the potential

218 for negative ecological effects, although these may be less than for traditional antibiotics.

220 An intriguing approach that may largely avoid the problem of system scale changes in

221 microbial community structure, as well as that of increasing antimicrobial resistance, is to

222 non-lethally target specific enzymes in the bacteria. This has been realized at the multi-

223 species level [57] through targeted inhibition of bacterial tri-methyl amine (TMA)

224 formation by 3,3-Dimethyl-1-butanol (DMB, a structural analog of choline) ultimately

225 attenuating atherosclerosis in a high choline diet mouse model. Surprisingly, slight

226 alterations of bacterial composition were still observed, underscoring the extremely

227 dynamic nature of the microbiome. Nonetheless, this study points towards a microbiome-

228 based intervention for a specific (i.e., "Western") diet-driven disease. In this case, a

229 single target approach is undesirable, as reduction of global TMA formation is the goal,

230 but given the availability of single isozyme inhibitors [58], precision, non-lethal drugs

231 likely could be developed. These furthermore have the potential to be minimally

232 bioavailable to the human, limiting side effects, and might be exploited not only to target 
233 pathogens but also to reduce microbiota-drug interactions through selective elimination 234 of problem microbes.

236 A final approach for targeted antimicrobials has been successfully employed for 237 approximately 100 years, though not as popularly in the western world [59]. Phages were 238 independently discovered in France and England, though developed as a therapy first in

239 the former. Despite great successes in treatment, especially of cholera, commercialization 240 of phage therapy failed due to production problems and other complications and so was 241 subsequently ignored in the US and Europe after the development of antibiotics [60].

242 Scientists in the Soviet Union (especially Georgia) continued to develop phage therapy, 243 having been cut off from antibiotic advances due to World War II. Here it was effectively 244 used it to control outbreaks of gastrointestinal diseases and refined further during the 245 Cold War and afterwards [61]. The basic premise of this technique is that many bacterial 246 species, and maybe even each strain (sub-species), are predated upon by a unique phage 247 [62]. Phage target bacteria cell-membrane protein and sugar complexes that are unique to 248 each bacterial taxon. Therefore, by identifying the correct phage it should be possible to 249 precisely remove a specific bacterial species from an assemblage. This will enable 250 accurate restructuring of a microbiome so as to precisely augment the functional 251 properties of that consortium. In fact, recent evidence from the commercial sector 252 suggests that the same mechanisms employed by phages to target and penetrate bacterial 253 cells can be programmed into nano-particles that mimic these phage-properties to infect 254 and kill specific cells (Pers. Comm. Jeffrey Miller, UCLA). In this new future, we may 255 have ultimate control over the microbiome. 


\section{Prebiotic treatments}

258 Conversely, instead of targeting the microbiome to reduce deleterious bacteria, one could

259 aim to increase the levels of beneficial bacteria or otherwise positively alter the structure

260 or function of the microbiome. Substances applied in this way are often referred to as

261 prebiotics. However, the types of prebiotics currently studied are limited in scope, usually

262 non-digestible fiber compounds that stimulate growth of Bifidobacterium and other taxa

263 to produce short chain fatty acids (SCFA) including butyrate and propionate [63].

264 Though this is promising as a broad treatments for several conditions [64], efforts for

265 precision medicine in this sphere will require the expansion of the scope of prebiotics.

266 Given that metagenomic and metabolomic advances continue to better characterize the

267 metabolic potential of the microbiome, especially across groups with vastly different

268 diets [65], prebiotic compounds that stimulate alternative beneficial bacteria towards

269 useful metabolic endpoints will be discovered [66].

271 More audaciously, one might aim at fine-tuning the interactions between microbiota of

272 the gut microbiome. The microbiome is a complex, human co-evolved ecosystem that

273 produces many bioactive compounds, often for intercellular communication [26]. These

274 compounds could be mined to find those which modulate the microbiome in a beneficial

275 way, thus unearthing novel prebiotics [67]. While microbial community disruption is the

276 consequence of both xenobiotic and microbiome targeted drug metabolism, these types of

277 prebiotics might provide a more gentle perturbation than possible with the former by

278 harnessing already existing biological pathways. This goal certainly seems distant, but as 
279 dynamical systems approaches to studying the microbiome continue to develop, we may

280 find that treating certain dysbiotic states require perturbations of varying magnitudes or

281 delicate maintenance of the stability of the microbiome, especially in at-risk populations $282[68]$.

283

284 Precision probiotics

285 Perhaps the most direct strategy for altering the microbiome is the usage of probiotics,

286 live microbes administered for health benefits. This idea has been employed since at least

2871907 when Élie Metchnikoff hypothesized lactic acid producing bacteria could implant in

288 the gastrointestinal tract to enhance longevity [69]. Today the probiotic landscape is still

289 dominated by lactic acid bacteria, specifically genera Lactobacillus, though it is now

290 appreciated that their beneficial properties are not limited to the production of a single

291 metabolite and that other potential probiotic bacteria, perhaps isolated from healthy

292 individuals [70], could affect various outcomes through multifarious means [71]. This

293 opens the door to precision probiotic development since application of microorganisms is

294 highly specific with regards to both applied agent and effect. Devices now exist for

295 isolating microorganisms based on metabolic output [72], and work is being done to

296 identify probiotic bacteria that produce particular compounds of therapeutic potential

297 [73]. This may include compounds whose efficacies are contingent on route of

298 administration, for example those that are inactive orally. Furthermore, probiotics are

299 being bioengineered to expand their ranges and modes of actions as well as their

300 robustness and incorporation [74]. However, it is important to keep in mind that

301 interactions with diet, established microbiota, and genetics, are known to modulate 
302 overall health outcomes if not specific effects and mechanisms of probiotics [71].

303 Therefore effective patient classification and stratification is required for best results.

304 Success of this program will require detailed insights into metagenomic potential and

305 ecological interactions of presumptive probiotic bacteria, making precision probiotic

306 development a task of considerable difficulty but one that has already seen demonstrable

307 results, for example in enhancing resistance to Clostridium difficile infection [75] and

308 suppressing hepatocellular carcinoma growth in mice [76].

\section{Regulation and Application}

311 Despite the therapeutic promise of the microbiome, its application to precision medicine 312 requires overcoming considerable hurdles. One may anticipate that failure to successfully 313 apply genomic medicine may lead to delays in the application of the microbiome as a

314 precision therapy. For example, the current legal and R\&D model is not well suited for 315 development of genome-informed drugs [77]. Microbiome therapies likewise face 316 difficulties, especially owing to the wide breadth of treatment options, many of which

317 lack analogs in current medical practice. Furthermore, clinicians have been reticent to use 318 the results of genomic information — and thus likely future microbiome data — in

319 treatment due to both uncertainties on its importance and lack of understanding [78]. 320 These problems are highlighted in the case of Plavix ${ }^{\circledR}$ (clopidogrel), whereby despite an 321 FDA box warning [79] indicating serious or fatal risk for those carrying certain 322 CYP2C19 variants, this drug is still routinely used on genetically incompatible patients 323 due to poor coverage by insurance and failure to clinically utilize genetic testing [80]. In 324 the case of the microbiome, fecal transplant treatment for Clostridium difficile colitis is 
325 known to be highly effective especially in recurrent infection [81]; however, this 326 procedure still requires a licensed practitioner to have a protocol approved by their local

327 Institutional Review Board, and therefore each patient needs to be consented prior to 328 therapy. For a therapy with $>90 \%$ success rate this is peculiar. However, it is because we 329 still lack the ability to characterize the microbial community of donor stool appropriately.

330 This means that we do not know the active components of the fecal transplant, and

331 therefore it is very difficult to regulate this using standard legislation under FDA

332 protocols. More importantly, we still don't fully understand the implications for

333 microbiome therapy on a large scale. While fecal transplants are becoming extremely

334 numerous with few legitimate side effects, it is still hard to predict the outcome across a

335 broad population. The same is true for genomic medicine, whereby the interaction of

336 genes with the environment is difficult to predict [82]. This requires enormous sample

337 populations for any investigation to be statistically significant [83]. Though the future is

338 bright for genomic medicine, particular issues currently impede efforts towards its

339 development.

341 Fortunately, some of the difficulties in genomic medicine research and deployment might

342 be lessened in precision microbiome medicine. Environmental-microbiome interactions

343 are potentially more easily studied because there is a more direct interaction between the

344 two, allowing for simpler identification of sample populations and achievement of

345 statistical power. With the correct experimental design, genetic variation can be

346 sufficiently decoupled from microbiome and environmental factors. In fact, studies of

347 this nature already exist, both on humans [84] and especially on mice, where genetics can 
348 be well controlled [85]. This bottom-up approach can then be extended by genomic

349 studies which better account for confounding factors. Even where genetics is a significant

350 factor, such as in mental health disorders, incorporating the microbiome greatly increases

351 understanding and ultimately treatment of diseases [86]. Of course in disease states where

352 the effects of genetic variation are either entirely or nearly absent, the microbiome is a

353 great candidate for investigation. Conditions such as obesity [87] and inflammatory

354 bowel disease [88] can in subsets of patients be driven by dysbiosis, a chronic, systemic

355 maladaptation of the gut microbiome to the host. Unlike genomic medicine, there are

356 possibilities especially for these conditions to do research in an in vitro environment,

357 most excitingly in artificial gut paradigms [89]. Microbiome precision medicine also has

358 the opportunity to break free of present $\mathrm{R} \& \mathrm{D}$ and legal hurdles to precision medicine.

359 The regulation and marketing of these treatments will at least pose challenges for

360 traditional models [90,91], as evidenced by the FDA's current stance on probiotics [92],

361 which has led to faster product delivery to the public but also quality control and

362 effectiveness issues [93]. Prioritizing treatments will be an important aspect of achieving

363 R\&D, FDA, and ultimately clinician support; unnecessary testing on low risk

364 communities and for low benefit interventions, will only hamper the development of

365 microbiome precision medicine.

366

\section{Notable Application: Medically Underserved Communities}

368 Given the above unique assets of the microbiome modality of precision medicine, a 369 promising potential area for its development is in low socio-economic status (SES) and 370 other under-served communities. Low SES is associated with reduced diversity in the gut 
371 microbiome [94]. Numerous factors are also present especially in urban communities that

372 reduce immunoregulation, including reduced exposure to microbes in the natural

373 environment [95] and increased stress [96], and increase obesity prevalence and

374 dysbiosis, including increased density of fast-food restaurants [97] and lack of physical

375 activity [98]. This is likely interrelated with microbiome-associated diseases such as

376 asthma [99] and gastrointestinal symptoms [100]. The vast majority of genomic variants

377 discovered are either rare with large effects or common with small effects, unlike in this

378 situation where there is a possibility of appreciable effect size combined with biomarker

379 occurrence. Therefore, these at-risk communities present a potentially illuminating cohort

380 for microbiome.

381

382 Of course, great care must be taken to not draw inappropriate or invalid associations 383 between microbiome [101] (or genome [102]) variations and minority status. Lack of 384 cultural understanding and disparities in access to services have driven poor research 385 trends in the past and continue to be a deep issue in the development of precision 386 medicine. Access issues in particular have caused demonstrable problems; statistics on 387 epidermal growth factor receptor testing, for example, show associations of lower 388 educational attainment and income with reduced likelihood of testing [103], and studies 389 suggest health insurance coverage alone does not explain this general effect $[104,105]$.

390 For precision medicine to succeed then, under-served populations must be both active 391 participants and beneficiaries of research. Microbiome research in particular could lead to 392 high impact clinical interventions for these communities, hopefully spurring its 393 development. It is both an opportunity and imperative for microbiome precision medicine 
394 to address social epidemiological trends, but this is only possible through the combined 395 efforts of researchers, clinicians, the government, and perhaps most importantly the 396 people at large.

\section{Concluding Remarks}

399 Here we have presented a collection of potential avenues towards introducing the 400 microbiome into precision medicine. Though it is difficult to know if and when these

401 techniques will ultimately make it to the clinic (see Outstanding Questions), there is 402 substantial evidence that microbiome-based medicine holds great future potential to 403 improve odds-ratios, reduce side effects, stratify patients, and precisely treat previously 404 difficult or untreatable conditions. Ultimately, the microbiome must become an integral 405 part of precision medicine as a whole, since so much of human functioning and 406 metabolism is dependent upon it. If this is to happen in the near future, as it hopefully 407 should, we must better understand the microbiome and its interactions with the human 408 and the environment via a concerted effort and conversation between researchers, 409 clinicians, patients, the government, and most importantly, the broader community.

\section{$411 \quad$ Figure 1}

412 A schematic of methods in precision microbiome medicine and their possible interplay:

413 a) As an example, certain microbes, here represented in red, metabolize the compound 414 cycasin to produce a carcinogenic compound methylazoxymethanol (MAM) [106]. This 415 functional potential of the microbe might be discovered through metagenomic 416 sequencing. b) If targeted removal of the red microorganism — identified in a patient via 
417 16S sequencing — was desired, without harming commensal bacteria, represented in 418 shades of blue, three approaches (green arrows) might be utilized. Direct removal of the 419 deleterious microorganism through targeted antibiotics ideally would not affect 420 commensal bacteria. Probiotic treatment introduces new beneficial microorganisms while 421 prebiotic treatment favors the growth of existing beneficial microorganisms. Note that 422 prebiotic and probiotic treatments do not directly remove the targeted microorganism, but

423 in certain cases may shift the gut ecology such that it does not thrive [107]. In all three 424 cases, the specific circumstances may affect which treatment is best employed and what 425 residual outcomes there are on the microbiome.

426

\section{References}

4281 Venter, J.C. et al. (2001) The sequence of the human genome. Science 291, 1304$429 \quad 51$

4302 Guttmacher, A.E. et al. (2002) Genomic Medicine - A Primer. N. Engl.J. Med. $431 \quad 347,1512-1520$

4323 McCarthy, J.J. et al. (2013) Genomic Medicine: A Decade of Successes, 433 Challenges, and Opportunities. Sci.Transl. Med. 5, 189sr4-189sr4

4344 Garraway, L.A. et al. (2013) Precision Oncology: An Overview. J. Clin. Oncol. $435 \quad 31,1803-1805$

4365 Schork, N.J. (2015) Personalized medicine: Time for one-person trials. Nature $437 \quad 520,609-611$

4386 Lederberg, J. (2000) Infectious history. Science 288, 287-293

4397 Sender, R. et al. (2016) Are We Really Vastly Outnumbered? Revisiting the Ratio 
440 of Bacterial to Host Cells in Humans. Cell 164, 337-340

4418 ElRakaiby, M. et al. (2014) Pharmacomicrobiomics: the impact of human

442 microbiome variations on systems pharmacology and personalized therapeutics.

$443 \quad$ OMICS $18,402-14$

4449 Surana, N.K. en Kasper, D.L. (2014) Deciphering the tete-a-tete between the

445 microbiota and the immune system. J. Clin. Invest. 124, 4197-4203

44610 Sampson, T.R. en Mazmanian, S.K. (2015) Control of Brain Development,

447 Function, and Behavior by the Microbiome. Cell Host Microbe 17, 565-576

44811 Dinan, T.G. et al. (2015) Collective unconscious: How gut microbes shape human

449 behavior. J. Psychiatr. Res. 63, 1-9

45012 Clarke, G. et al. (2014) Minireview: Gut Microbiota: The Neglected Endocrine

$451 \quad$ Organ. Mol. Endocrinol. 28, 1221-1238

45213 (2010) Human genome at ten: The sequence explosion. Nature 464, 670-671

45314 Franzosa, E.A. et al. (2015) Identifying personal microbiomes using metagenomic

$454 \quad$ codes. Proc. Natl. Acad. Sci. 112, E2930-E2938

45515 David, L.A. et al. (2013) Diet rapidly and reproducibly alters the human gut

456 microbiome. Nature 505, 559-563

45716 Gilbert, J.A. et al. (2016) Microbiome-wide association studies link dynamic

458 microbial consortia to disease. Nature $535,94-103$

45917 Clarridge, J.E. en Alerts, C. (2004) Impact of 16S rRNA gene sequence analysis

460 for identification of bacteria on clinical microbiology and infectious diseases. Clin.

$461 \quad$ Microbiol. Rev. 17, 840-862

46218 Woo, P.C.Y. et al. (2008) Then and now: Use of 16S rDNA gene sequencing for 
463 bacterial identification and discovery of novel bacteria in clinical microbiology

$464 \quad$ laboratories. Clin. Microbiol. Infect. 14, 908-934

46519 Galley, J.D. et al. (2014) Maternal obesity is associated with alterations in the gut

466 microbiome in toddlers. PLoS One 9,

46720 Sharpton, T.J. (2014) An introduction to the analysis of shotgun metagenomic

$468 \quad$ data. Front. Plant Sci. 5, 209

46921 Sangwan, N. et al. (2016) Differential Functional Constraints Cause Strain-Level

470 Endemism in Polynucleobacter Populations. mSystems 1, e00003-16

47122 Cardona, C. et al. (2016) Network-based metabolic analysis and microbial

$472 \quad$ community modeling. Curr. Opin. Microbiol. 31, 124-131

47323 Thomas, T. et al. (2012) Metagenomics - a guide from sampling to data analysis.

474 Microb. Inform. Exp. 2, 3

47524 Jeffery, I.B. et al. (2012) Categorization of the gut microbiota: enterotypes or

476 gradients? Nat. Rev. Microbiol. 10, 591-2

$47725 \quad$ Faust, K. et al. (2015) Metagenomics meets time series analysis: unraveling

478 microbial community dynFaust, K., Lahti, L., Gonze, D., de Vos, W. M., \& Raes,

479 J. (2015). Metagenomics meets time series analysis: unraveling microbial

480 community dynamics. Current Opinion in Microbiology. Curr. Opin. Microbiol.

$481 \quad 25,56-66$

48226 Donaldson, G.P. et al. (2015) Gut biogeography of the bacterial microbiota. Nat.

$483 \quad$ Rev. Microbiol. 14, 20-32

48427 Rowan, F. et al. (2010) Bacterial Colonization of Colonic Crypt Mucous Gel and

485 Disease Activity in Ulcerative Colitis. Ann. Surg. 252, 869-875 
48628 Kalow, W. en Genest, K. (1957) A METHOD FOR THE DETECTION OF

487 ATYPICAL FORMS OF HUMAN SERUM CHOLINESTERASE.

488 DETERMINATION OF DIBUCAINE NUMBERS. Biochem. Cell Biol. 35, 339-

$489 \quad 346$

49029 Sultana, J. et al. (2013) Clinical and economic burden of adverse drug reactions. J.

$491 \quad$ Pharmacol. Pharmacother. 4, 73

49230 Chan, A.L.F. et al. (2008) Cost evaluation of adverse drug reactions in

493 hospitalized patients in Taiwan: A prospective, descriptive, observational study.

$494 \quad$ Curr. Ther. Res. 69, 118-129

49531 Edwards, I.R. en Aronson, J.K. (2000) Adverse drug reactions: definitions,

496 diagnosis, and management. Lancet 356, 1255-1259

49732 Verbeurgt, P. et al. (2014) How common are drug and gene interactions?

498 Prevalence in a sample of 1143 patients with CYP2C9, CYP2C19 and CYP2D6

499 genotyping. Pharmacogenomics 15, 655-665

50033 Guengerich, F.P. (2008) Cytochrome P450 and Chemical Toxicology. Chem. Res.

$501 \quad$ Toxicol. $21,70-83$

50234 Sousa, T. et al. (2008) The gastrointestinal microbiota as a site for the

503 biotransformation of drugs. Int. J. Pharm. 363, 1-25

50435 R. Rizkallah, M. et al. (2012) The PharmacoMicrobiomics Portal: A Database for

505 Drug-Microbiome Interactions. Curr. Pharmacogenomics Person. Med. 10, 195-

$506 \quad 203$

50736 Carmody, R.N. en Turnbaugh, P.J. (2014) Host-microbial interactions in the

508 metabolism of therapeutic and diet-derived xenobiotics. J. Clin. Invest. 124, 4173- 
$509 \quad 4181$

51037 Wilson, I.D. en Nicholson, J.K. (2015) The Modulation of Drug Efficacy and

$511 \quad$ Toxicity by the Gut Microbiome. bll 323-341

51238 Rautio, J. et al. (2008) Prodrugs: design and clinical applications. Nat. Rev. Drug

$513 \quad$ Discov. 7, 255-270

51439 Watkins, P.B. et al. (2006) Aminotransferase Elevations in Healthy Adults

515 Receiving 4 Grams of Acetaminophen Daily. JAMA 296, 87

51640 Clayton, T.A. et al. (2009) Pharmacometabonomic identification of a significant

517 host-microbiome metabolic interaction affecting human drug metabolism. Proc.

$518 \quad$ Natl. Acad.Sci.U.S. A. 106, 14728-14733

51941 Swanson, H.I. (2015) Drug Metabolism by the Host and Gut Microbiota: A

520 Partnership or Rivalry? Drug Metab. Dispos. 43, 1499-1504

52142 Takasuna, K. et al. (1996) Involvement of beta-glucuronidase in intestinal

522 microflora in the intestinal toxicity of the antitumor camptothecin derivative

523 irinotecan hydrochloride (CPT-11) in rats. Cancer Res. 56, 3752-7

52443 Haiser, H.J. en Turnbaugh, P.J. (2013) Developing a metagenomic view of

$525 \quad$ xenobiotic metabolism. Pharmacol. Res. 69, 21-31

52644 Shajib, M.S. en Khan, W.I. (2015) The role of serotonin and its receptors in

527 activation of immune responses and inflammation. Acta Physiol. 213, 561-574

52845 Viaud, S. et al. (2013) The intestinal microbiota modulates the anticancer immune

$529 \quad$ effects of cyclophosphamide. Science 342, 971-6

53046 Davey, K.J. et al. (2013) Antipsychotics and the gut microbiome: olanzapine-

531 induced metabolic dysfunction is attenuated by antibiotic administration in the rat. 
$532 \quad$ Transl. Psychiatry 3, e309

53347 Worsley, M. a (1998) Infection control and prevention of Clostridium difficile

534 infection. J Antimicrob Chemother 41 Suppl C, 59-66

53548 Rubinstein, E. en Camm, J. (2002) Cardiotoxicity of fluoroquinolones. $J$.

$536 \quad$ Antimicrob. Chemother. 49, 593-6

53749 Galatti, L. et al. (2005) Neuropsychiatric reactions to drugs: an analysis of

538 spontaneous reports from general practitioners in Italy. Pharmacol. Res. 51, 211-6

$53950 \quad$ Rubin, B.K. en Tamaoki, J., reds (2005) Antibiotics as Anti-Inflammatory and

$540 \quad$ Immunomodulatory Agents, Birkhäuser-Verlag.

54151 Bailey, M.T. et al. (2011) Exposure to a social stressor alters the structure of the

542 intestinal microbiota: Implications for stressor-induced immunomodulation. Brain.

543 Behav. Immun. 25, 397-407

54452 Wallace, B.D. en Redinbo, M.R. (2013) The human microbiome is a source of

$545 \quad$ therapeutic drug targets. Curr. Opin. Chem. Biol. 17, 379-384

54653 Maurice, C.F. et al. (2013) Xenobiotics shape the physiology and gene expression

547 of the active human gut microbiome. Cell 152, 39-50

54854 Eckert, R. et al.(2006) Targeted killing of Streptococcus mutans by a pheromone-

549 guided "smart" antimicrobial peptide. Antimicrob. Agents Chemother. 50, 3651-7

55055 Guo, L. et al. (2015) Precision-guided antimicrobial peptide as a targeted

551 modulator of human microbial ecology. Proc. Natl. Acad. Sci. U. S. A. 112, 7569-

$552 \quad 7574$

55356 Belda-Ferre, P. et al. (2015) The human oral metaproteome reveals potential

554 biomarkers for caries disease. Proteomics $15,3497-507$ 
55557 Wang, Z. et al. (2015) Non-lethal Inhibition of Gut Microbial Trimethylamine

556 Production for the Treatment of Atherosclerosis. Cell 163, 1585-1595

55758 Yao, J. et al. (2016) A Pathogen-Selective Antibiotic Minimizes Disturbance to the

558 Microbiome. Antimicrob. Agents Chemother. DOI: 10.1128/AAC.00535-16

55959 Nobrega, F.L. et al. (2015) Revisiting phage therapy: new applications for old

$560 \quad$ resources. Trends Microbiol. 23, 185-191

56160 Kutter, E. et al. (2010) Phage Therapy in Clinical Practice: Treatment of Human

562 Infections. Curr. Pharm. Biotechnol. 11, 69-86

56361 Summers, W.C. (2012) The strange history of phage therapy. Bacteriophage 2,

$564 \quad 130-133$

56562 Koskella, B. en Meaden, S. (2013) Understanding Bacteriophage Specificity in

$566 \quad$ Natural Microbial Communities. Viruses 5, 806-823

56763 Petschow, B. et al. (2013) Probiotics, prebiotics, and the host microbiome: The

568 science of translation. Ann. N. Y. Acad. Sci. 1306, 1-17

56964 Candela, M. et al.(2010) Functional intestinal microbiome, new frontiers in

$570 \quad$ prebiotic design. Int. J. Food Microbiol. 140, 93-101

57165 O'Keefe, S.J.D. et al. (2015) Fat, fibre and cancer risk in African Americans and

$572 \quad$ rural Africans. Nat. Commun. 6, 6342

57366 Preidis, G.A. en Versalovic, J. (2009) Targeting the Human Microbiome With

574 Antibiotics, Probiotics, and Prebiotics: Gastroenterology Enters the Metagenomics

$575 \quad$ Era. Gastroenterology 136, 2015-2031

57667 Garber, K. (2015) Drugging the gut microbiome. Nat. Biotechnol. 33, 228-231

57768 Gerber, G.K. (2014) The dynamic microbiome. FEBS Lett. 588, 4131-4139 
57869 Gordon, S. (2008) Elie Metchnikoff: Father of natural immunity. Eur. J. Immunol.

$579 \quad 38,3257-3264$

58070 Belda-Ferre, P. et al. (2012) The oral metagenome in health and disease. ISME J.

$581 \quad 6,46-56$

58271 Bron, P. a. et al. (2011) Emerging molecular insights into the interaction between

583 probiotics and the host intestinal mucosa. Nat. Rev. Microbiol. 10, 66-78

58472 Gavrish, E. et al. Devices and methods for the selective isolation of

585 microorganisms. . (2016), Google Patents

58673 Strandwitz, P. et al. GABA-Modulating Bacteria : Microbiome-Based

$587 \quad$ Therapeutics for Depression? , RISE. (2015)

58874 Amalaradjou, M.A.R. en Bhunia, A.K. (2013) Bioengineered probiotics, a

589 strategic approach to control enteric infections. Bioengineered 4, 379-387

59075 Buffie, C.G. et al. (2014) Precision microbiome reconstitution restores bile acid

591 mediated resistance to Clostridium difficile. Nature 517, 205-8

59276 Li, J. et al. (2016) Probiotics modulated gut microbiota suppresses hepatocellular

593 carcinoma growth in mice. Proc. Natl. Acad.Sci. U. S. A. 113, E1306-15

59477 Thompson, B.M. en Boiani, J. (2015) The Legal Environment for Precision

595 Medicine. Clin. Pharmacol. Ther. [Accepted, 167-169

59678 Ginsburg, G.S. (2013) Realizing the opportunities of genomics in health care.

597 JAMA 309, 1463-4

59879 Bristol-Myers Squibb/Sanofi Pharmaceuticals Partnership Plavix [package insert].

$599 \quad .(2015), 1-32$

60080 Johnson, J.A. et al. (2012) Clopidogrel: a case for indication-specific 
601 pharmacogenetics. Clin. Pharmacol. Ther. 91, 774-6

60281 van Nood Els et al. (2013) Duodenal Infusion of Donor Feces for Recurrent

603 Clostridium difficile. N. Engl.J.Med. 368, 407-415

60482 Manuck, S.B. en McCaffery, J.M. (2014) Gene-Environment Interaction. Annu.

$605 \quad$ Rev. Psychol. 65, 41-70

60683 Gauderman, W.J. (2002) Sample size requirements for matched case-control

607 studies of gene-environment interaction. Stat. Med. 21, 35-50

60884 Huang, Y.J. en Boushey, H.A. (2015) The microbiome in asthma. J. Allergy Clin.

$609 \quad$ Immunol. $135,25-30$

61085 Ellekilde, M. et al. (2014) Transfer of gut microbiota from lean and obese mice to 611 antibiotic-treated mice. Sci.Rep. 4,

61286 Foster, J. a. en McVey Neufeld, K.A. (2013) Gut-brain axis: How the microbiome 613 influences anxiety and depression. Trends Neurosci. 36, 305-312

61487 Turnbaugh, P.J. et al. (2006) An obesity-associated gut microbiome with increased 615 capacity for energy harvest. Nature 444, 1027-31

61688 Frank, D.N. et al. (2007) Molecular-phylogenetic characterization of microbial 617 community imbalances in human inflammatory bowel diseases. Proc. Natl. Acad.

$618 \quad$ Sci. 104, 13780-13785

61989 Shah, P. et al. (2016) A microfluidics-based in vitro model of the gastrointestinal 620 human-microbe interface. Nat. Commun. 7, 11535

62190 Sun, X. et al. (2016) Patent watch: Modulating the human microbiome with live 622 biotherapeutic products: intellectual property landscape. Nat. Rev. Drug Discov. $623 \quad 15,224-225$ 
62491 Morgan, B. (2016) Drug development: A healthy pipeline. Nature 533, S116-S117

62592 Degnan, F.H. (2008) The US Food and Drug Administration and Probiotics:

626 Regulatory Categorization. Clin. Infect. Dis. 46, S133-S136

62793 Lewis, Z.T. et al. (2016) Validating bifidobacterial species and subspecies identity

628 in commercial probiotic products. Pediatr. Res. 79, 445-452

62994 Miller, G.E. et al. (2016) Lower Neighborhood Socioeconomic Status Associated

630 with Reduced Diversity of the Colonic Microbiota in Healthy Adults. PLoS One

$631 \quad 11, \mathrm{e} 0148952$

63295 Rook, G.A.W. et al. (2014) Microbial “old friends”, immunoregulation and

633 socioeconomic status. Clin. Exp. Immunol. 177, 1-12

63496 Bailey, M.T. et al. (2011) Exposure to a social stressor alters the structure of the

635 intestinal microbiota: Implications for stressor-induced immunomodulation. Brain.

636 Behav. Immun. 25, 397-407

63797 Block, J.P. et al. (2004) Fast food, race/ethnicity, and income: A geographic

$638 \quad$ analysis. Am. J. Prev.Med.27, 211-217

63998 Powell, L.M. et al. (2006) Availability of Physical Activity-Related Facilities and

640 Neighborhood Demographic and Socioeconomic Characteristics: A National

641 Study. Am. J. Public Health 96, 1676-1680

64299 Almqvist, C. et al. (2005) Low socioeconomic status as a risk factor for asthma,

643 rhinitis and sensitization at 4 years in a birth cohort. Clin. Exp. Allergy 35, 612-8

644100 Bytzer, P. (2001) Low socioeconomic class is a risk factor for upper and lower

645 gastrointestinal symptoms: a population based study in 15000 Australian adults.

$646 \quad$ Gut $49,66-72$ 
647101 McGuire, A.L. et al. (2008) Ethical, legal, and social considerations in conducting

648 the Human Microbiome Project. Genome Res. 18, 1861-1864

649102 Qureshi, N. en Kai, J. (2005) Genomic medicine for underserved minority

650 populations in family medicine. Am. Fam. Physician 72, 386-7

651103 Lynch, J.A. et al. (2013) Utilization of epidermal growth factor receptor (EGFR)

652 testing in the United States: a case study of T3 translational research. Genet. Med.

$653 \quad 15,630-638$

654104 Adler, N.E. (1993) Socioeconomic Inequalities in Health. JAMA 269, 3140

655105 Penson, D.F. et al. (2001) The association between socioeconomic status, health

656 insurance coverage, and quality of life in men with prostate cancer. J. Clin.

$657 \quad$ Epidemiol. 54, 350-358

658106 Spatz, M. et al.(1967) Role of intestinal microorganisms in determining cycasin

659 toxicity. Proc. Soc. Exp. Biol. Med. 124, 691-7

660107 Schoeni, J.L. en Wong, A.C. (1994) Inhibition of Campylobacter jejuni

661 colonization in chicks by defined competitive exclusion bacteria. Appl. Environ.

$662 \quad$ Microbiol. 60, 1191-7

663 


\section{Outstanding Questions}

What is the relative significance of specific microbial actors versus whole microbiome ecology in disease states, and how will drugging specific bacteria affect ecological succession following this perturbation? How will this depend on the milieu in which a species is situated (e.g., presence of different taxa performing a similar ecological role)? Additionally, what roles might phages, fungi, viruses play?

How closely coupled are genetics and the microbiome, and how can these fields be integrated into a unified practice of precision medicine?

Which microbiome-driven disease states can be successfully cured? Which instead require prophylactic or palliative, noncurative therapy?

What is the best way to move precision microbiome medicine results out into the clinic? What changes in regulatory, governmental as well as research and development processes will need to occur for this to happen?

How will the needs of different groups be best addressed across diets, lifestyles, and environments? What interventions will ultimately require social change rather than medical therapy, and what will the interplay between these fields be? 
Figure ${ }^{1}$ Metagenomic insight

(B) 165 assay
and diagnosis

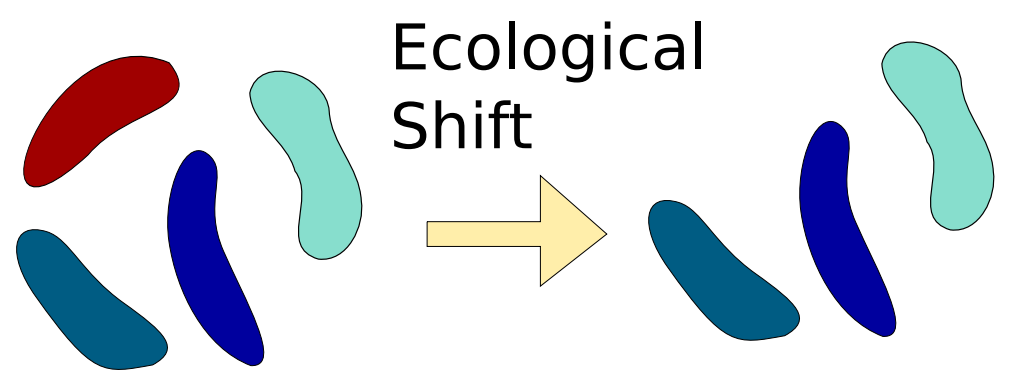

Cycasin

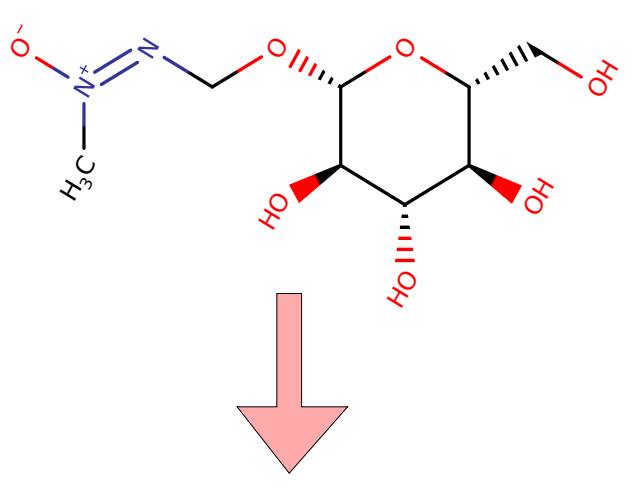

Probiotic

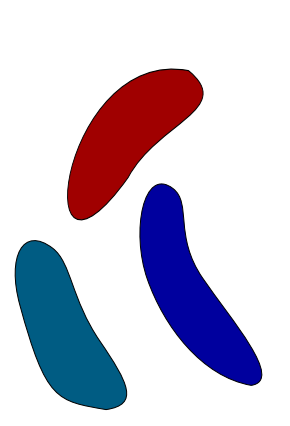

Targeted antibiotic
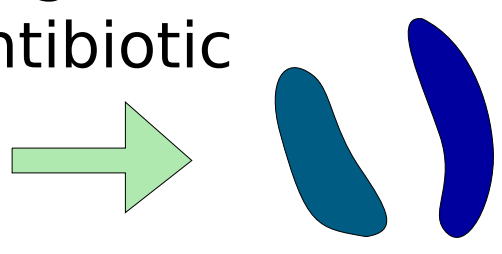

Prebiotic

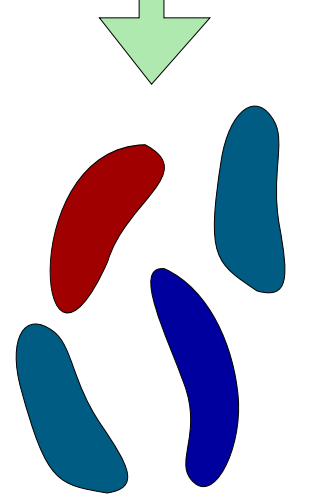

Ecological Shift

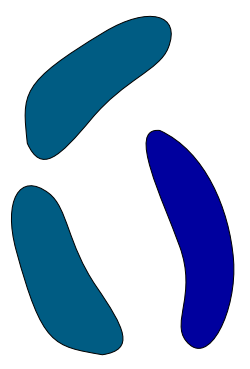

